\title{
Nano-antenna-assisted harmonic generation
}

\author{
Nils Pfullmann • Christian Waltermann - Milutin Kovačev • Vanessa Knittel • \\ Rudolf Bratschitsch • Dieter Akemeier • Andreas Hütten • Alfred Leitenstorfer • \\ Uwe Morgner
}

\begin{abstract}
We report on low-order harmonic generation utilising the plasmonic field enhancement in arrays of rodtype gold optical antennae. Furthermore, we examine their suitability to support high-order harmonic generation (HHG). The low-order harmonics are used as a tool to investigate the nonlinear properties of the antennae. Particular attention is paid to the thermal properties, which become significant at the peak intensities necessary for HHG. A theoretical model explains the experimental findings and enables future improvements. In experiments we observe up to the fifth harmonic order and measure a field enhancement sufficient to support high-order harmonic generation. Moreover, we find a damage threshold for the antennae.
\end{abstract}

N. Pfullmann $(\bowtie) \cdot$ C. Waltermann · M. Kovačev $\cdot$ U. Morgner Institut für Quantenoptik, Leibniz Universität Hannover, Welfengarten 1, 30167 Hannover, Germany

e mail: pfullmann@iqo.uni hannover.de

N. Pfullmann · C. Waltermann · M. Kovačev · U. Morgner QUEST, Centre for Quantum Engineering and Space Time Research, Hannover, Germany

V. Knittel · A. Leitenstorfer

Department of Physics, Center for Applied Photonics,

University of Konstanz, 78457 Konstanz, Germany

R. Bratschitsch

Institut für Physik, Dynamik nanoskopischer und

mesoskopischer Strukturen, Technische Universität Chemnitz, 09107 Chemnitz, Germany

D. Akemeier · A. Hütten

Thin Films and Physics of Nanostructures, Department of

Physics, Bielefeld University, 35501 Bielefeld, Germany

U. Morgner

Laser Zentrum Hannover e.V., Hannover, Germany

\section{Introduction}

For radio frequencies and in the microwave regime, antennae are widely used for various applications. In the visible spectral range, however, the concept has only recently been introduced [1]. Optical antennae offer a wide range of applications though, ranging from the extreme concentration and manipulation of light [2], nonlinear optics [3] to the generation of high-order harmonic radiation [4]. This process is of particular interest because it paves the way for experiments on attosecond time-scales [5], e.g. among other things, new measurements in fundamental physics to study electron dynamics in molecules. Moreover, it enables the extension of the frequency comb technique [6] in high-precision spectroscopy to the ultraviolet regime. However, its extension to this spectral range is challenging due to the low conversion efficiency of the HHG process. To circumvent this issue and to increase the harmonic photon flux, external enhancement cavities have been developed and investigated extensively [ 710$]$. By employing a plasmonic resonance in optical nano-antennae, an alternative approach for HHG directly from a laser oscillator is envisaged [4]. Various challenges such as atomic line emission [11] also make this technique complicated. A successful implementation of this novel scheme, however, would bridge the gap between two different fields of physics: plasmonics with typically low pulse energies and structure dimensions in the nanometre regime on one hand and high-field physics with peak intensities in excess of $10^{14} \mathrm{~W} \mathrm{~cm}^{-2}$ on the other.

Here, we present a detailed analysis of rod-type optical antennae with respect to their suitability to support HHG. Low-order harmonics are used as a tool to investigate the nonlinear properties of the antennae. Particular attention is paid to the thermal properties, which become significant at 
the peak intensities necessary for HHG. Using a theoretical model, we are able to explain the experimental findings, giving rise for future improvements.

\section{Experimental setup}

Figure 2 depicts the experimental setup. A Ti:sapphire oscillator (Venteon, Pulse one) delivers pulses with a pulse duration of $15 \mathrm{fs}$ and a pulse energy of up to $5 \mathrm{~nJ}$ centred at $825 \mathrm{~nm}$. The pulses are negatively chirped by several bounces on dispersive mirrors (DCM) to compensate for dispersion added later, i.e. a vacuum window, an achromatic lens, and the substrate. The dispersion is fine-tuned via a pair of thin fused silica wedges. Finally, a telescope with a ratio of 1:3 expands the beam size to enable tight focussing with a variety of achromatic lenses. The lens is mounted on a translation stage to adjust the focal position as indicated by the green arrow, without breaking the vacuum. The focal spot size ranges from $12.4 \times 8.2$ to $8.2 \times 5.6 \mu \mathrm{m}$ with corresponding peak intensities of $2.5 \times 10^{11}$ to $8.1 \times 10^{11} \mathrm{~W} \mathrm{~cm}^{-2}$, respectively. The sample with the nano-antennae is attached to a second translation stage moving in $x$ - and $y$-direction to selectively address different antenna arrays, which appear as disks in the SEM image in Fig. 1 due to their extremely high packing density. The laser polarisation and the antenna axis are aligned parallel to each other. To efficiently detect the generated harmonic radiation a confocal monochromator setup (modified McPherson 234/302, 1,200 lines/mm grating) is used, where the generation volume acts as an entrance slit. The sample is thus mounted in the focal point of the toroidal mirror and the photon collection efficiency is maximised [12, 13]. Finally, a PMT capable of single photon counting (Hamamatsu H8259-09) is used in combination with a photon counter (Scientific Research SR400) to measure the third harmonic signal behind the exit slit.
By flipping a mirror into the harmonic beam and setting the monochromator to the appropriate wavelength, it is possible to easily switch between signal detection for the third harmonic and higher harmonic orders. The latter are detected with an electron multiplier (Photonis 4751G) instead of the PMT.

The rod-type gold nano-antennae are manufactured with electron beam lithography (EBL) on either a fused silica, a crystalline quartz or sapphire substrate with a thin chromium layer underneath to serve as an adhesion layer. The antennae are $25 \mathrm{~nm}$ wide and $120180 \mathrm{~nm}$ long with a thickness of $30 \mathrm{~nm}$ and a nominal gap size of $20 \mathrm{~nm}$, i.e. it varies by $\pm 5 \mathrm{~nm}$ at most. Typical differences are much smaller and in the order of $2 \mathrm{~nm}$. The arrays have a circular shape with a diameter of $25 \mu \mathrm{m}$ as can be seen in the SEM image inset in Fig. 1 or in the third harmonic map in Fig. 2. A detailed SEM picture of the antennae prior to illumination with the laser beam is shown in Fig. 6a.

We use the third harmonic from the nano-antennae to optimise the focal position as well as the alignment of the antenna array relative to the laser focus. High vacuum is necessary for the propagation of higher harmonic orders. During the experiments the vacuum chamber is therefore kept at a background pressure of $1 \times 10^{-6} \mathrm{mbar}$.

\section{Results and discussion}

\subsection{Low-order harmonics}

A strong third harmonic signal is measured when the laser focus is aligned to a nano-antenna array as expected from other experiments [3,14]. Figure 2 shows an image, where the laser focus has been systematically scanned across an antenna array. On the antenna array the third harmonic emission is significantly enhanced with respect to the surrounding material. Moreover, inhomogeneities within
Fig. 1 Experimental setup. Laser pulses from a Ti:sapphire oscillator are focused by an achromatic lens onto arrays of rod antennae. Different arrays are located on the sample and appear as disks in the small SEM image inset. The zoomed image depicts a single array with a diameter of $25 \mu \mathrm{m}$. Low harmonic orders are generated and analysed with different detectors (see text for details)

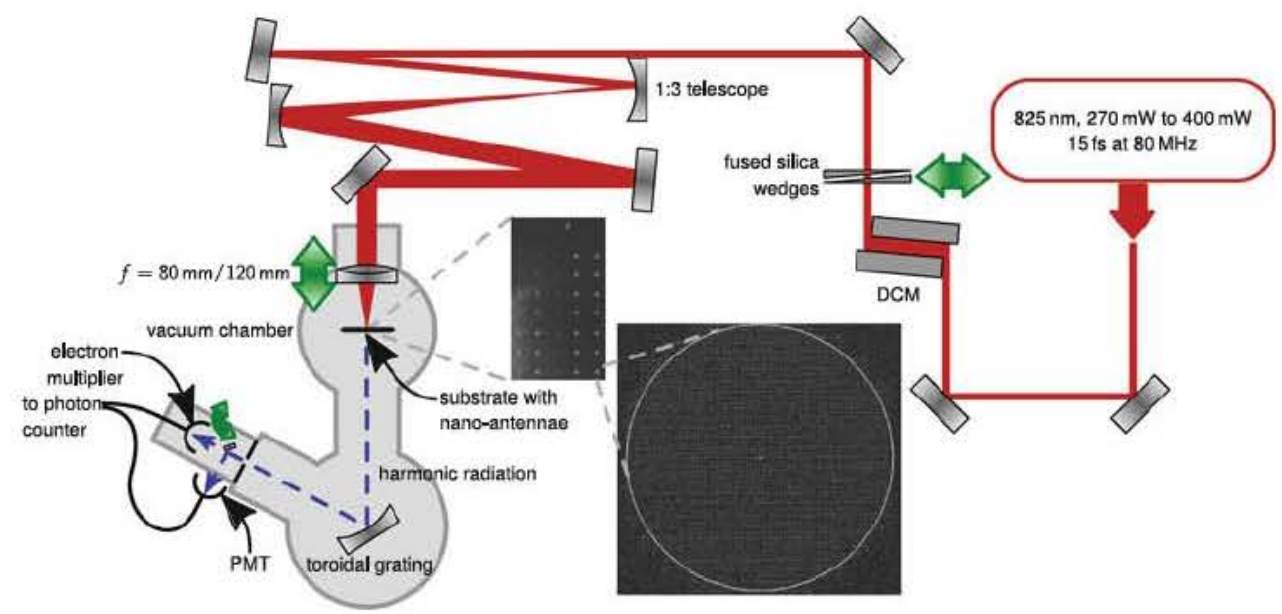




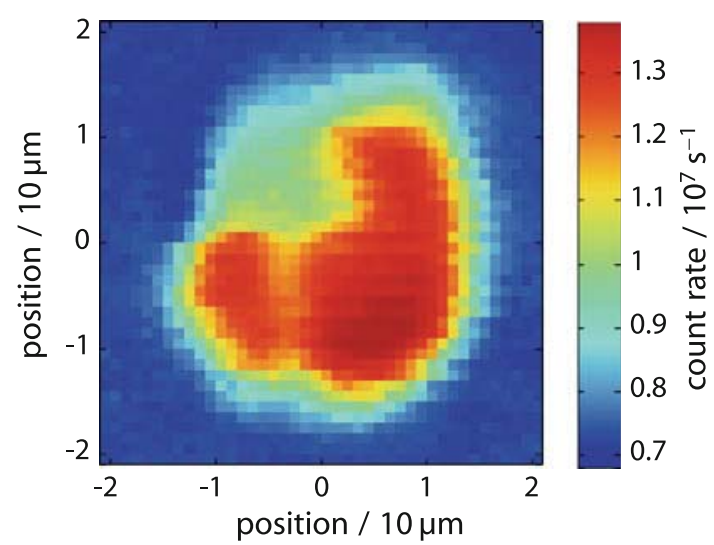

Fig. 2 Third harmonic map of an antenna array. Significant enhance ment occurs on the antenna array and inhomogeneities due to antenna destruction become apparent. Only the upper left part of the array was illuminated with an intense laser beam leading to the observed partial destruction

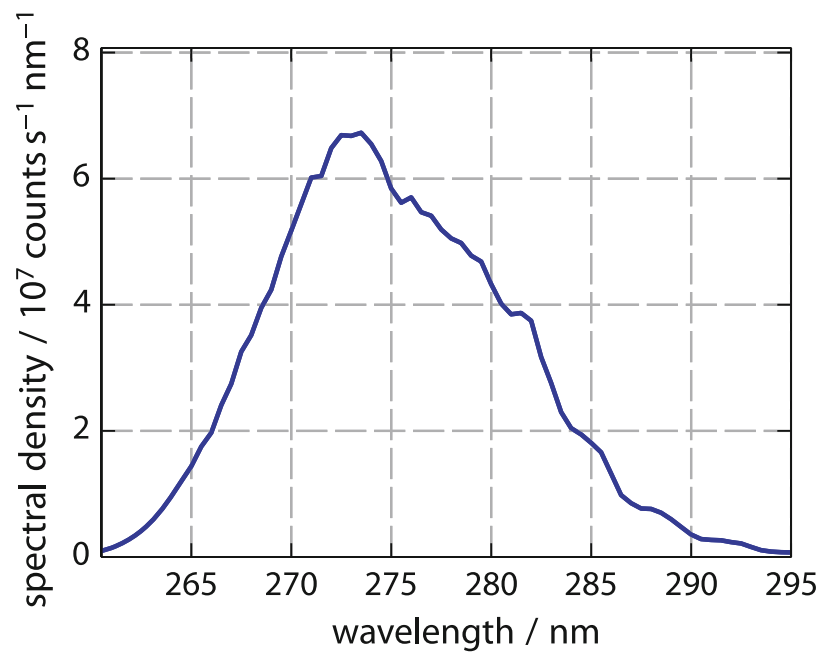

Fig. 3 Third harmonic radiation generated from rod type optical antennae. The central wavelength is in perfect accordance with the wavelength of the driving laser of $825 \mathrm{~nm}$

the array become apparent. For example, the antennae in the upper left corner exhibit a lower enhancement, which is caused by antenna destruction as described later. The intensity enhancement factor in the gap region of the antennae has experimentally been determined to be in the order of $10^{2}$ by comparing the third harmonic signal from the antenna arrays with that of the bare substrate. A typical spectrum of the emitted third harmonic radiation is depicted in Fig. 3 with the central wavelength matching perfectly to the wavelength of the driving laser of $825 \mathrm{~nm}$. By scanning the laser focus along the $z$-direction, it was confirmed that the third harmonic is generated at the interface between the substrate and the ambient vacuum. Additionally to the third harmonic, we also observe the

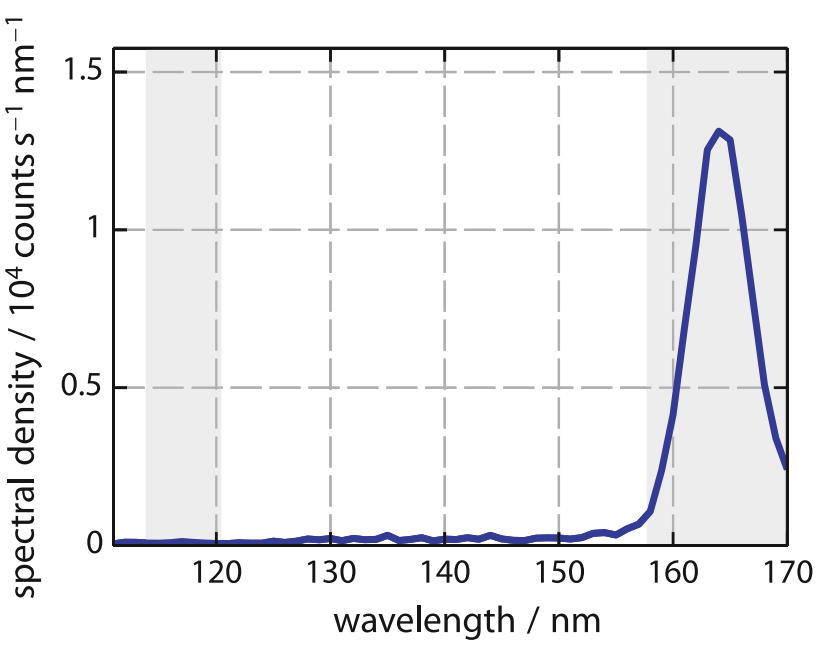

Fig. 4 Fifth harmonic radiation generated from the rod type optical antennae. Grey background shading indicates the spectral positions of the harmonic orders. The width of the shading is determined from the fundamental laser spectrum

fifth harmonic plotted in Fig. 4. The spectrum is corrected for the detection efficiency set by the grating and the electron multiplier and was taken under vacuum conditions with a background pressure of $1 \times 10^{-6}$ mbar. The harmonic orders of the driving laser are indicated by grey background shading, with the fifth harmonic located at $165 \mathrm{~nm}$ and the seventh at $118 \mathrm{~nm}$. The width of the shading is determined from the fundamental laser spectrum. A pronounced peak is measured at the fifth harmonic, which reveals a ratio of $10^{-4}$ in comparison with peak signals from the earlier THG measurements. A significant decrease is expected from theoretical calculations and also agrees with earlier results [15]. Due to the symmetry of the system only odd harmonics are generated; and consequently, no even harmonics are observed in Fig. 4. Moreover, the harmonic signal originates from the surface as no gas was fed onto the sample. More importantly, it confirms the resonant enhancement of the incident electric field and thus the feasibility of high-order harmonic generation.

\subsection{Thermal stability}

Figure 5 shows the harmonic signal at $164 \mathrm{~nm}$ as well as an exponential fit as a function of the illumination time. We observe an exponential decrease of the harmonic signal, which saturates at about $40 \%$ of its initial value and increases again after translating the sample to illuminate a fresh spot within the antenna array. Despite the substantial decrease, the remaining enhancement of the nano-antennae is still sufficient to support low-order harmonic generation. The decrease of the harmonic signal is caused by melting of the nano-antennae as depicted in the SEM images in Fig. 6. Figure 6a shows the antennae prior to illumination 


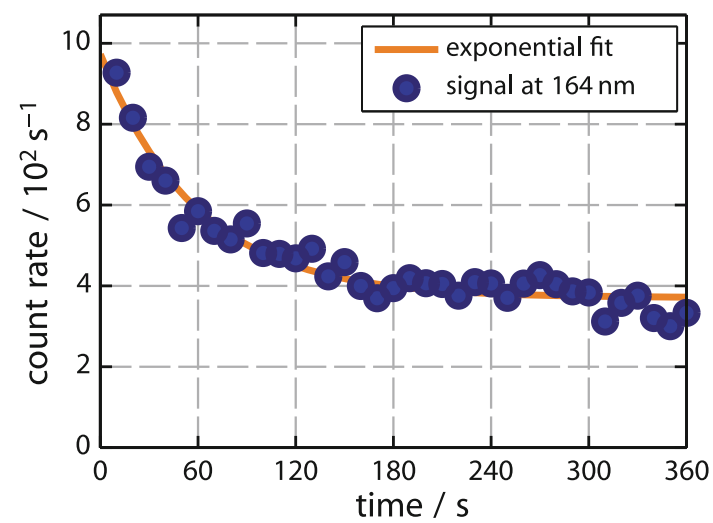

Fig. 5 Fifth harmonic signal with respect to illumination time as well as an exponential fit. An exponential decrease is observed, which saturates at approx. $40 \%$ of its initial value. Significant enhancement remains to further support low order harmonic generation (see text for details)

(a)

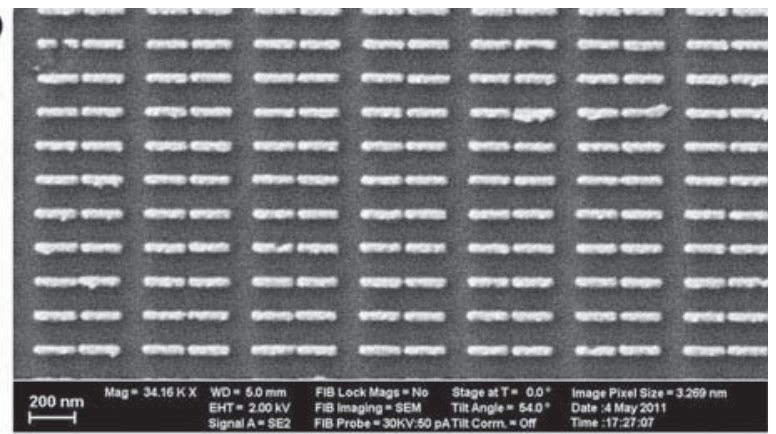

(b)

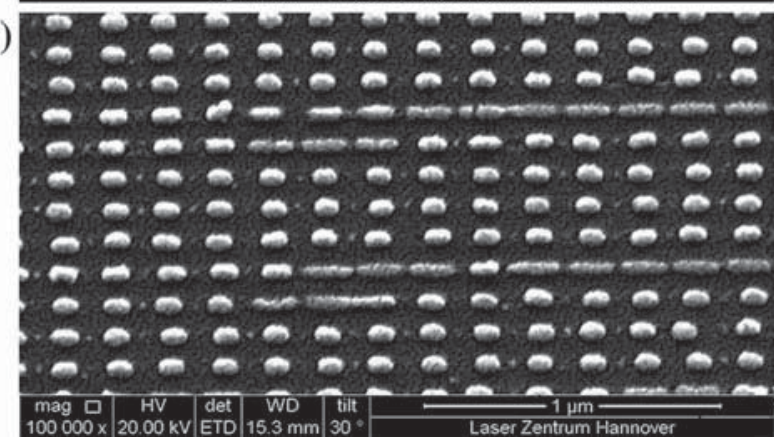

Fig. 6 SEM images of rod type optical antennae before (a) and after (b) illumination with the laser pulses. Melting is a major damage process as shown in $\mathbf{b}$

with the laser beam with clearly identifiable rod-type optical antennae separated by a gap. After illumination however, the antennae have melted into pronounced blobs which are visible in Fig. 6b. This also leads to a significantly increased gap size and hence a reduced electric field enhancement. Consequently, the nonlinear signal is also reduced. A similar destruction with a dramatically reduced nonlinear signal or even the complete absence has recently also been reported by Park et al. [16] in a similar experiment. At the incident intensities this behaviour is
Table 1 Physical constants of used metals and substrate materials [18]

\begin{tabular}{lllll}
\hline & Gold & Sapphire & $\begin{array}{l}\text { Fused } \\
\text { silica }\end{array}$ & Unit \\
\hline Thermal conductivity, $\kappa$ & 317 & 42 & 1.38 & $\mathrm{~W} \mathrm{~m}^{-1} \mathrm{~K}^{-1}$ \\
Specific heat capacity, $c$ & 129 & 750 & 1,050 & $\mathrm{~J} \mathrm{~kg}^{-1} \mathrm{~K}^{-1}$ \\
Mass density, $\rho$ & 19.32 & 3.97 & 2.2 & $\times 10^{3} \mathrm{~kg} \mathrm{~m}^{-3}$ \\
Thermal diffusivity, $a$ & 127 & 4.7 & 0.199 & $\times 10^{-6} \mathrm{~m}^{2} \mathrm{~s}^{-1}$ \\
Melting temperature & 1,336 & 2,313 & 1,988 & $\mathrm{~K}$ \\
Heat of fusion & 62.8 & & & $\times 10^{3} \mathrm{~J} \mathrm{~kg}^{-1}$ \\
\hline
\end{tabular}

Table 2 Further simulation parameters

\begin{tabular}{lcl}
\hline Parameters & Values & Unit \\
\hline Peak intensity & 28 & $\times 10^{11} \mathrm{~W} \mathrm{~cm}^{-2}$ \\
Pulse duration & 15 & $\mathrm{fs}$ \\
Antenna volume & 9 & $\times 10^{-23} \mathrm{~m}^{3}$ \\
Heat source density & 318 & $\mathrm{~J} \mathrm{~m}^{-3}$ \\
\hline
\end{tabular}

understood by a simple theoretical model presented in the following.

\subsection{Theoretical model}

By solving the diffusion equation and taking the heat of fusion of gold into account, the temperature increase of the nano-antennae is calculated for successive laser pulses on different substrate materials. All relevant material parameters are given in Table 1. The respective laser and antenna parameters are taken from the experiment and are listed in Table 2. Only one pair of antennae is considered in the calculation and as a first approximation, the exact temperature distribution in the antenna is neglected and a spatially averaged heat source density is used. Moreover, heat diffusion from the antenna into the substrate material is the predominant cooling process due to the orders of magnitude larger thermal conductivity of, e.g. sapphire or fused silica in comparison to the surrounding medium. Solving the diffusion equation is therefore reduced to a one dimensional problem, which can be implemented much more easily [17].

Figure 7 shows the calculated temperature for a gold antenna deposited on a fused silica substrate. As expected, the antenna experiences a steep temperature increase after illumination with a single laser pulse. Subsequently, the antenna cools down due to heat diffusion into the substrate. This cooling scheme is primarily governed by the thermal conductivity of the substrate material and substantially faster cooling occurs in case of a sapphire substrate. Nonetheless, even for fused silica the antenna cools down 


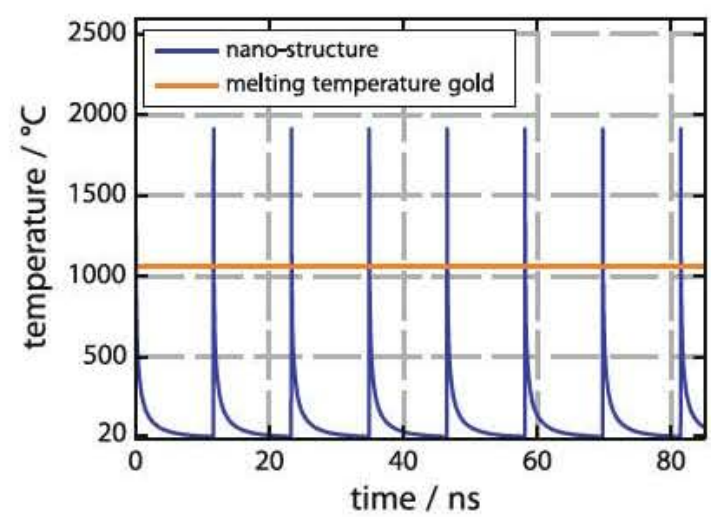

Fig. 7 Temperature evolution for a gold nano antenna on a fused silica substrate at an incident intensity of $5 \times 10^{11} \mathrm{~W} \mathrm{~cm}^{-2}$. The melting temperature of gold is clearly exceeded shortly after illumination with a laser pulse

to their initial temperature before illumination with the next pulse. Heat accumulation can therefore be ruled out as the source of antenna destruction. On the contrary, already shortly after the laser pulse hits the antenna, the melting temperature of gold is clearly exceeded and melting of the antenna becomes a likely damage process. Experimentally we found a damage threshold for the antennae between $2.5 \times 10^{11}$ and $5.4 \times 10^{11} \mathrm{~W} \mathrm{~cm}^{-2}$, which was reproduced by the simple model giving an intensity of $3.6 \times 10^{11} \mathrm{~W} \mathrm{~cm}^{-2}$. It can therefore be used to find thermally more stable antenna parameters.

\section{Conclusion}

In conclusion, we have shown that rod-type nano-antennae manufactured with electron beam lithography facilitate an intensity enhancement in the gap region in the order of $10^{2}$.
Low harmonic orders are observed in the experiment which demonstrate the level of field enhancement. Currently melting of the antennae limits further applications at the incident intensities, which are aimed to generate high-order harmonics via an interaction with noble gases in the gap region. A simple theoretical model is in agreement with the experimentally found results. It therefore allows the determination of thermally more stable antenna parameters by incorporating thermally induced damage into the design process.

Acknowledgments This work was funded by Deutsche Fors chungsgemeinschaft within the Cluster of Excellence QUEST, Centre for Quantum Engineering and Space Time Research.

\section{References}

1. P. Mühlschlegel, H. J. Eisler, O.J.F. Martin, B. Hecht, D.W. Pohl, Science 308, 1607 (2005)

2. J.A. Schuller et al., Nat. Mater. 9, 193 (2010)

3. T. Hanke et al., Phys. Rev. Lett. 103, 257404 (2009)

4. S. Kim et al., Nature 453, 757 (2008)

5. F. Krausz, M. Ivanov, Rev. Mod. Phys. 81, 163 (2009)

6. T. Udem, R. Holzwarth, T. W. Hänsch, Nature 416, 233 (2002)

7. C. Gohle et al., Nature 436, 234 (2005)

8. R.J. Jones, K.D. Moll, M.J. Thorpe, J. Ye, Phys. Rev. Lett. 94, 193201 (2005)

9. A. Ozawa et al., Phys. Rev. Lett. 100, 253901 (2008)

10. D.C. Yost et al., Nat. Phys. 5, 815 (2009)

11. M. Sivis, M. Duwe, B. Abel, C. Ropers, Nature 485, E1 (2012)

12. X.F. Li, A. L'Huillier, M. Ferray, L.A. Lompré, G. Mainfray, Phys. Rev. A 39, 5751 (1989)

13. F. Lindner et al., Phys. Rev. A 68, 013814 (2003)

14. T. Hanke et al., Nano Lett. 12, 992 (2012)

15. T. Tsang, Phys. Rev. A 54, 5454 (1996)

16. I. Y. Park et al., Annalen Phys. 525, 87 (2013)

17. G. Baffou, H. Rigneault, Phys. Rev. B 84, 035415 (2011)

18. W. Benenson, J.W. Harris, H. Stocker, H. Lutz, eds., Handbook of Physics, (Springer, Berlin, 2000) 